\title{
Presencia de Pseudomonas aeruginosas, cepas BLEE Y RESISTENCIA EN SALAS DEL HOSPITAL SOLOMON KLEIN, COCHABAMBA
}

\author{
Presence of Pseudomonas Aeruginosas, BLEE strains AND \\ RESistance in ROOMS OF the SOlOMON KLEIN HOSPITAL, COCHABAMBA
}

Danny Junior Pascual Araoz', Adriana Belén Flores Ocampo', Andrea Camila Quiroga Pérez', Brenda Almendras Terrazas', Katheryne Romaneth Crespo Osinaga².

\section{RESUMEN}

Las infecciones causadas por Pseudomona aeruginosa y cepas BLEE junto con la resistencia bacteriana, producto del uso exagerado e inapropiado de los antibióticos, han favorecido al aumento importante de microorganismos resistentes a múltiples fármacos por lo que se ha convertido en un problema importante de salud de difícil manejo. Objetivo: Conocer la presencia de Pseudomona aeruginosa y cepas BLEE junto con su resistencia en la Sala de Maternidad y Neonatología del Hospital Solomon Klein el año 2019. Materiales y Métodos: Estudio de tipo descriptivo y transversal, se incluyó porta-sueros, agarrador de las camas, bandeja del material de curación, llave de los sueros, mangueras de los sueros e incubadoras para tomar muestras de estos. Resultados: Se tomaron 25 muestras de la sala de maternidad y neonatología de las que 3 (I2\%) resultaron positivos a Pseudomona aeruginosa en superficies de porta sueros, agarrador de camilla y bandeja de material de curación del servicio de Enfermería de la Sala de Maternidad. En las 21 (84\%) muestras restantes prevaleció la presencia de la enterobacteria Hafnia alvei, en el análisis de resistencia resultaron sensibles a Cefotaxima descartando la existencia de cepas BLEE en el hospital. Discusión: las infecciones asociadas a servicios de salud ocurren principalmente durante el ingreso y estancia hospitalaria y es por eso que la vigilancia de estos patrones facilita conocer los mecanismos de acción predominantes de los servicios, lo cual, es muy importante al momento de tomar decisiones terapéuticas.

\section{ABSTRACT}

Infections caused by Pseudomona aeruginosa and BLEE strains together with the bacterial resistance due to exaggerated and incorrect use of antibiotics have favored the increase in the importance of microorganisms resistant to multiple drugs, which has become an important health problem for difficult handling Objective: To know the presence of Pseudomona aeruginosa and BLEE strains together with their resistance in the Maternity and Neonatology Room of the Solomon Klein Hospital in 2019.

Materials and Methods: Descriptive study and cross-sectional type include serum-holder, grabber the beds, tray of the healing material, key of the sera, hoses of the sera and incubators to take samples of these. Results: We took 25 samples from the maternity and neonatology ward of which 3 (12\%) were positive for Pseudomona aeruginosa on serum carrier surfaces, stretcher gripper and Cure Material Tray of the Nursing Service of the Maternity ward . In the 2 I (84\%) remaining samples, the presence of Hafnia alvei enterobacteria prevailed, in the resistance analysis they were sensitive to Cefotaxime, ruling out the existence of BLEE strains in the hospital. Discussion: infections associated with health services occur mainly during hospital admission and hospital stay and that is why monitoring these patterns makes it easier to know the predominant mechanisms of action of the services, which is very important at the moment.of making therapeutic decisions.

\section{INTRODUCCIÓN}

$P^{\text {eudomona aeruginosa es una bacteria Gram }}$

Pnegativa oportunista, no fermentadora de glucosa y aerobia facultativa, caracterizada por su capacidad de proliferar en medios anaerobios utilizando nitratos o arginina en lugar de oxígeno como aceptor para los electrones; la resistencia bacteriana de este agente puede estar dada por la presencia de B-lactamasas y carbapenemasas ${ }^{1}$. Cuenta con la capacidad de elaborar una gran diversidad de factores de virulencia entre los cuales incluye adhesinas, toxinas, enzimas entre otros ${ }^{2,3}$; por lo tanto, el pronóstico de la enfermedad dependerá de la ubicación que ocupe el patogeno ${ }^{4}$.
'Estudiante de Tercer Año de la Facultad de Medicina, Universidad Mayor de San Simón

Correspondencia a: Nombre: Danny Junior Pascual Araoz.

Correo electrónico: nacionalcalama2014@gmail.com Telf. y celular: +59170376829 ORCID: https://orcid.org/00000002-8643-0284

https://orcid.org/0000-00019220-3494

https://orcid.org/0000-00019342-6569

https://orcid.org/0000-00024230-9300

https://orcid.org/0000-00015979-4499

Palabras clave: Farmacorresistencia, Pseudomona aeruginosa, Beta-Lactamasas.

Keywords: Drug resintance, Pseudomonas aeruginosa, Beta-Lactamases.

Procedencia y arbitraje: no comisionado, sometido a arbitraje externo.

Recibido para publicación: 4 de octubre de 2019 Aceptado para publicación: 14 de noviembre de 2020

Citar como:

Pascual Araoz DJ, Flores Ocampo AB, Quiroga Pérez AC, Almendras Terrazas B, Crespo Osinaga KR. Presencia de Pseudomonas aeruginosas, cepas BLEE y resistencia en Salas del Hospital Solomon Klein, Cochabamba. Rev Cient Cienc Med 2020; 23(2): 129-135

Presencia, resistencia de Pseudomona aeruginosa 
La familia de enterobacterias está asociada a infecciones intrahospitalarias, de las cuales cobran mayor importancia las cepas productoras de b-lactamasas de espectro extendido (BLEE), como Escherichia coli y Klebsiella pneumoniae, microorganismos que se han encontrado en gran cantidad en áreas de enfermería, áreas de limpieza, baños, salas quirúrgicas, neonatales y unidades de cuidado intensivo produciendo focos de infección para la adquisición de infecciones nosocomiales ${ }^{6,7}$.

Las infecciones por Pseudomona aeruginosa suelen manifestarse a nivel cutáneo, en heridas, por la facilidad del patógeno de proliferar en zonas húmedas, asociándose a bacteriemias ${ }^{8}$. Las manifestaciones gastrointestinales representan e indican gravedad y mal pronóstico clínico. En lactantes prevalecen las alteraciones gastrointestinales, ectima gangrenosa y shock séptico 9 .

Las infecciones en el tracto urinario y la diarrea, mayormente se deben a $\mathcal{E}$. coli, en cambio infecciones relacionadas con neumonías y sepsis intrahospitalarias, corresponde a Klebsiella pneumoniae, representando mayor riesgo en neonatos ${ }^{10}$.

La resistencia a los desinfectantes por parte de $\mathscr{P}$. aeruginosa, se debe a la composición de su pared celular, en especial su membrana externa la cual actúa como una barrera que evita la entrada de un gran número de agentes bactericidas ${ }^{11}$. Los desinfectantes más eficaces contra $\mathcal{P}$. aeruginosa en hospitales, han sido los compuestos de amonio cuaternario, caracterizados por lesionar la integridad de la membrana celular ${ }^{12}$.

La prevalencia de Enterobacterias resistentes está en aumento en varios hospitales a nivel mundial, en especial patógenos como E. coli y $K$. pneumoniae ${ }^{13}$. Varios estudios de multirresistencia en enterobacterias, resaltan la producción de carbapenemasas, que en los últimos años ha alcanzado dimensiones preocupantes en distintos países, situación que imposibilita su uso en el tratamiento ${ }^{14}$. Los únicos beta-lactámicos efectivos frente a estas enterobacterias son las cefamicinas, la cefoxitina y las combinaciones de beta-lactámicos con inhibidores de beta-lactamasas ${ }^{15}$.

\section{MATERIALES Y MÉTODOS}

El presente estudio es de tipo descriptivo y transversal realizado en la ciudad de Cochabamba el Hospital de Segundo Nivel Salomón Klein, en julio 2019 cuyo universo está conformado por los ambientes de las Salas de Maternidad y Neonatología; tomando como muestra superficies con mayor probabilidad de contaminación, como ser: porta-sueros, agarrador de las camas, bandeja del material de curación, llave de los sueros, mangueras de los sueros e incubadoras.

Criterios de inclusión: Objetos con mayor manipulación por parte del personal de salud como también por parte de los pacientes y que están en contacto con estos cada día.

Criterios de exclusión: Componentes de la intubación, endoscopios y oxímetros.

Parala recolección delas muestras seutilizaron 25 hisopos estériles y 25 tubos de ensayo preparados con agar Pseudomona, enumerados según los lugares de la recolección de muestras, este agar fue preparado con anterioridad en el laboratorio de la Facultad de Medina de la Universidad Mayor de San Simón, una vez realizado el hisopado para la toma las muestra, se procedió a sembrarlo en los tubos de ensayo con el agar Pseudomona, medio selectivo que cuenta con los compuestos necesarios para el aislamiento de estos microorganismos, las muestras recogidas se trasladaron en ambiente debidamente refrigerado hacia el laboratorio de bacteriología de la facultad de medicina de la UMSS donde se prepararon y sembraron las muestras para luego refrigerar y esperar 24 a $48 \mathrm{hrs}$ para el crecimiento de las colonias; los hisopos utilizados se desecharon siguiendo las medidas de bioseguridad correspondientes.

De las muestras obtenidas tres dieron positivo para $\mathcal{P}$. aeruginosa; con las demás colonias se procedió a realizar la tinción de Gram para identificar la morfología microscópica de las bacterias, posteriormente se realizó la Prueba Bioquímica de Identificación de Enterobacterias, utilizando el agar Triple Sugar Iron, Lisina Hierro Agar, Simmons Citrate Agar, SIM Medium, B. A. M. médium en los cuales los marcadores de glucosa, lactosa y formadores de gas que resultaron positivos confirmando la presencia de enterobacterias, a continuación se procedió con las pruebas para identificar el tipo específico de enterobacterias, las pruebas que se realizaron, fueron las de: indol, lisina descarboxilasa, ácido sulfhídrico, 
citrato, urea y movilidad, dando positivo para algunos y negativo para otros, estos datos lo comparamos con una tabla de diferenciación de enterobacterias que mediante descarte identificamos al que tenía más semejanza con los datos de la tabla.

Para la prueba de perfil antimicrobiano se realizó la inserción de los discos de resistencia los cuales fueron medidos y comprobados según los parámetros del Clinical and Laboratory Stands Institute (CLSI), mediante el cual se determinaron cepas sensibles de acuerdo con la concentración mínima inhibitoria.

\section{RESULTADOS}

Pasadas las 24 horas de incubación se evidencio el crecimiento de colonias, donde se obtuvo un resultado positivo para Pseudomona aeruginosa en Agar Pseudomona en 3 de las 25 muestras, (Ver figura 1) las cuales fueron tomadas en la Sala de Maternidad y Neonatología en las superficies de: Agarrador de camilla de la Sala GOB-211 del Servicio de Maternidad, Porta sueros de la Sala P-207 de la Sala de Maternidad y de la Bandeja de material de Curación del Servicio de Enfermería de la Sala de Maternidad, obteniendo el $12 \%$ de las muestras recolectadas con resultado positivo.

La muestra Nro. 16 llevada a cabo en una de las Mangueras de Sueros del Servicio de Neonatología no se evidencio la presencia de

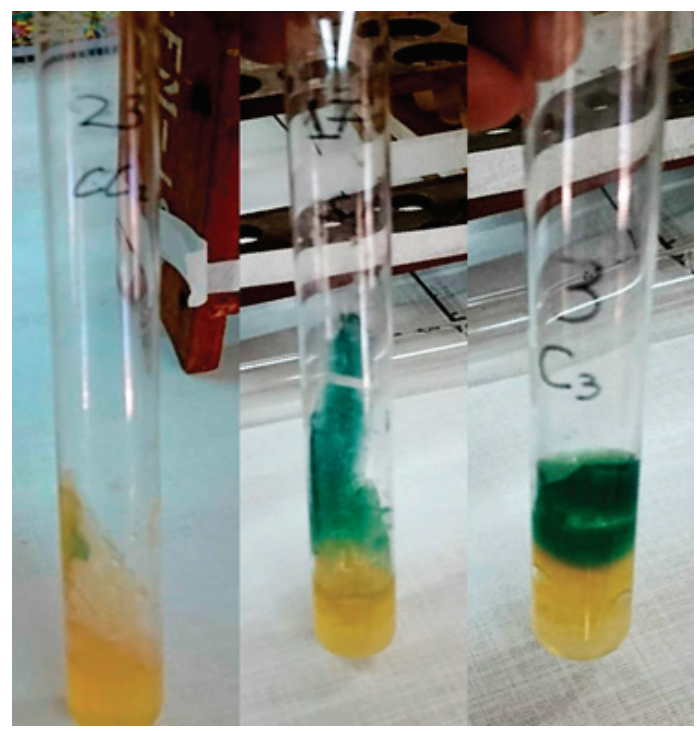

Figura 1. Presencia confirmada de colonias de Pseudomona aeruginosa
Pseudomona aeruginosa ni de enterobacterias.

En las 21 muestras restantes se identificaron enterobacterias a través de una serie química para enterobacterias. (Ver tabla-1). Analizando los resultados de las 25 muestras, se pudo evidenciar que la enterobacteria Hafnia alvei es la que más prevalece en las superficies en las que se llevó a cabo el estudio. (Ver Tabla-1).

El resultado del antibiograma realizado en las muestras positivas de Pseudomona aeruginosa evidenció que estas bacterias son sensibles a múltiples medicamentos. Ver tabla-2 y Figura 2).

En las muestras restantes que dieron positivos para enterobacterias se pudo evidenciar a través de un cultivo en agar MacConkey que todas fueron sensibles a Cefotaxima dando a descartar la presencia de cepas BLEE en el Hospital Solomon Klein.

\section{DISCUSIÓN}

E. coli es un microorganismo muy frecuentementeimplicadoenbacteriemiastanto comunitarias como nosocomiales, es necesario que se confirme e informe la producción de cepas BLEE con su previa identificación, esto debido al riesgo que corren los pacientes al

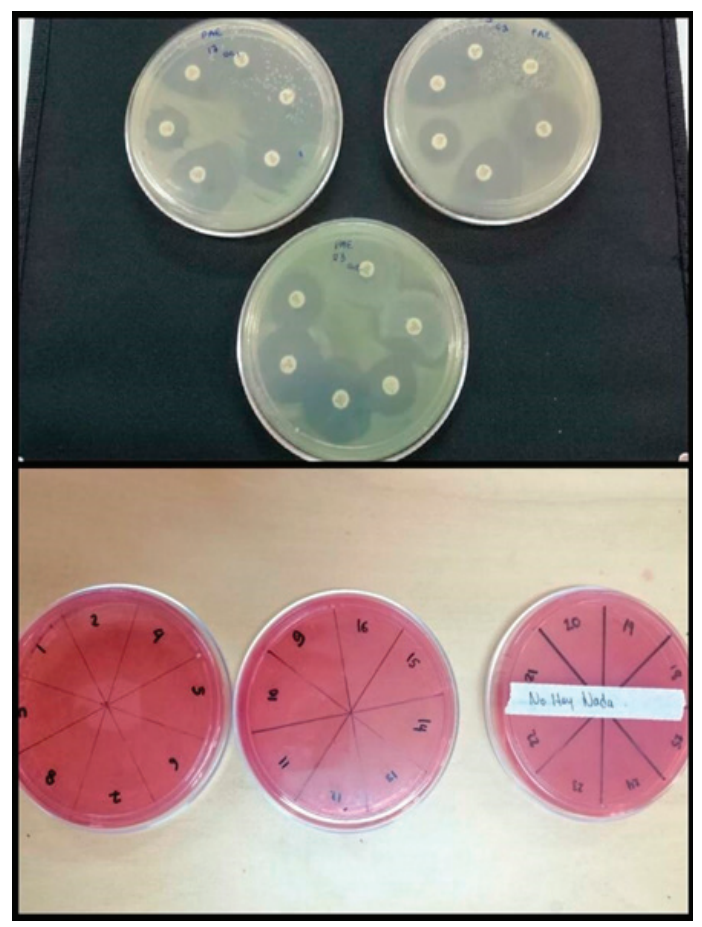

Figura 2. Antibiograma de Pseudomonas y

Enterobacterias. 
Tabla 1. Identificación de bacterias en Agarrador de Camillas y Porta sueros

\begin{tabular}{|c|c|c|c|}
\hline MUESTRAS & SALA & $\begin{array}{l}\text { SERIE QUIMICA PARA } \\
\text { ENTEROBACTERIAS }\end{array}$ & PORCENTAJES \\
\hline 1 Camilla 1 & GOB-203 & Klebsiella aerogenes & H. alvei $=44 \%$ \\
\hline 2 Camilla 2 & GOB-211 & Escherichia coli & E. coli $=28 \%$ \\
\hline 4 Camilla 4 & M-201 & Hafnia alvei & P. aeruginosa $=12 \%$ \\
\hline 5 Camilla 5 & M-201 & Hafnia alvei & K. aerogenes $=8 \%$ \\
\hline 6 Camilla 6 & M-201 & Kle6siella aerogenes & K. pneumoniae $=4 \%$ \\
\hline 7 P. sueros 1 & M-201 & Klebsiella pneumoniae & Sin resultado $=4 \%$ \\
\hline 8 P. sueros 2 & M-201 & Hafnia alvei & \\
\hline 9 P. sueros 3 & PED-207 & Hafnia alvei & \\
\hline 11 P. sueros 5 & IRAS 208 & Escherichia coli & \\
\hline 12 Llave de S. 1 & Neonatología & Hafnia alvei & \\
\hline 13 Llave de S. 2 & Neonatología & Hafnia alvei & \\
\hline 14 Llave de S. 3 & PED-207 & Escherichia coli & \\
\hline 15 Llave de S. 4 & PED-207 & Escherichia coli & \\
\hline 17 M. de suero 2 & Neonatología & Escherichia coli & \\
\hline 18 M. de suero 3 & PED-207 & Escherichia coli & \\
\hline 19 M. de suero 4 & PED-207 & Escherichia coli & \\
\hline 20 M. de suero 5 & IRAS 208 & Hafnia alvei & \\
\hline 21 Incubadora 1 & Neonatología & Hafnia alvei & \\
\hline 22 Incubadora 2 & Neonatología & Hafnia alvei & \\
\hline 23 Incubadora 3 & Neonatología & Hafnia alvei & \\
\hline 24 Bandeja 1 & Neonatología & Hafnia alvei & \\
\hline
\end{tabular}

FUENTE: elaboración propia. Esta tabla muestra los resultados de las muestras que dieron positivos para enterobacterias, identificando el género y la especie de cada una de ellas.

fallo de tratamiento. La distribución de estas cepas no es estática, por el contrario ha estado sujeta a una expansión constante; el European Antimicrobial Surveillance System (EARSS) encargado de monitorizar las resistencias antibióticas en patógenos invasivos desde 1998, ha mostrado en Europa (2006-2008) un aumento en la frecuencia de $\mathcal{E}$. coli resistente a cefalosporinas de $3^{\text {a }}$ generación ${ }^{16}$, es por esta razón que las bacterias gramnegativas tienen que ser constantemente controladas y monitoreadas por los centros de salud.

Las infecciones intrahospitalarias son un problema de salud por las implicaciones económicas sociales, es por eso que para su tratamiento se ha planteado un estricto control constantemente actualizado y vigilado del uso de antibióticos para evitar la resistencia bacteriana ${ }^{17}$; además de la importancia que conlleva saber que una infección cruzada exógena puede ser causada por objetos contaminados por el paciente y/o miembros del personal ${ }^{18}$. Tal como se observa en los resultados obtenidos de las superficies en las que fueron halladas las muestras de $\mathcal{P}$. Aeruginosa, se nos sugiere que los objetos sujetos a manipulación y a un deficiente control sanitario facilita la ocurrencia de las infecciones. 
Tabla 2. Perfil antimicrobiano de Pseudomonas y enterobacterias en superficies identificadas

\begin{tabular}{|c|c|c|c|c|c|}
\hline MUESTRA & BACTERIA & $\begin{array}{c}\text { MEDICAMEN- } \\
\text { TOS }\end{array}$ & CONT. & HALO & RESISTENCIA \\
\hline \multirow[t]{6}{*}{ 3-camilla 3} & $\begin{array}{c}\text { Pse u d o m o na } \\
\text { aeruginosa }\end{array}$ & Cefepime & 30 ug & $30 \mathrm{~mm}$ & Sensible \\
\hline & & $\begin{array}{c}\text { Piperacilin-ta- } \\
\text { zobactam }\end{array}$ & 110 ug & $25 \mathrm{~mm}$ & Sensible \\
\hline & & Imipenem & 10 ug & $19 \mathrm{~mm}$ & Sensible \\
\hline & & Ceftazidime & 30 ug & $25 \mathrm{~mm}$ & Sensible \\
\hline & & Ciprofloxacino & 5 ug & $39 \mathrm{~mm}$ & Sensible \\
\hline & & Gentamicina & $10 \mathrm{ug}$ & $20 \mathrm{~mm}$ & Sensible \\
\hline \multirow[t]{7}{*}{$10-P$. sueros 4} & $\begin{array}{c}\text { P se u d o mo n a } \\
\text { aeruginosa }\end{array}$ & & & & \\
\hline & & Cefepime & $30 \mathrm{ug}$ & $38 \mathrm{~mm}$ & Sensible \\
\hline & & $\begin{array}{c}\text { Piperacilin-ta- } \\
\text { zobactam }\end{array}$ & $110 \mathrm{ug}$ & $27 \mathrm{~mm}$ & Sensible \\
\hline & & Imipenem & 10 ug & $20 \mathrm{~mm}$ & Sensible \\
\hline & & Ceftazidime & $30 \mathrm{ug}$ & $22 \mathrm{~mm}$ & Sensible \\
\hline & & Ciprofloxacino & 5 ug & $36 \mathrm{~mm}$ & Sensible \\
\hline & & Gentamicina & 10 ug & $24 \mathrm{~mm}$ & Sensible \\
\hline \multirow[t]{7}{*}{ 25-Bandeja 2} & $\begin{array}{c}\text { P s e u d o m o n a } \\
\text { aeruginosa }\end{array}$ & & & & \\
\hline & & Cefepime & $30 \mathrm{ug}$ & $30 \mathrm{~mm}$ & Sensibilidad \\
\hline & & $\begin{array}{c}\text { Piperacilin-ta- } \\
\text { zobactam }\end{array}$ & 110 ug & $28 \mathrm{~mm}$ & Sensibilidad \\
\hline & & Meropenem & $10 \mathrm{ug}$ & $20 \mathrm{~mm}$ & Sensibilidad \\
\hline & & Ceftazidime & $30 \mathrm{ug}$ & $30 \mathrm{~mm}$ & Sensibilidad \\
\hline & & Ciprofloxacino & 5 ug & $28 \mathrm{~mm}$ & Sensibilidad \\
\hline & & Gentamicina & 10 ug & $23 \mathrm{~mm}$ & Sensibilidad \\
\hline Enterobacterias & & Cefotaxima & 4 ug & $0 \mathrm{~mm}$ & Sensibilidad \\
\hline
\end{tabular}

FUENTE: elaboración propia. Esta tabla muestra los resultados del perfil antimicrobiano, realizado con las muestras que dieron positivo para Pseudomona aeruginosa y enterobacterias, demostrando sensibilidad hacia los medicamentos utilizados en la investigación.

Se han registrado en el año 2002 un total de 1,7 millones de infecciones intrahospitalarias y casi 99.000 muertes relacionadas con estas bacterias; la presencia y la resistencia bacteriana es variable entre instituciones de salud o incluso una misma ciudad, por ejemplo: en Hospitales de Estados Unidos han sido identificadas las Bacterias Gram negativas de la familia Enterobacteriaceae productoras de carbapenemasas así como en otro lugares como China e inclusive Sudamérica y con menos frecuencia en Europa. La relación genética de las cepas responsables de estas infecciones resalta la importancia del control de su propagación y la decisión terapéutica; a fin de prevenir la morbimortalidad ${ }^{19}$.
En el tratamiento, ya sea de forma combinada o como monoterapia, se cuenta con las fluoroquinolonas, betalactámicos y cefalosporinas de 3ra generación que han sido utilizados como agentes que cuentan con actividad contra las bacterias gramnegativas en infecciones intrahospitalarias graves; sin embargo la selección de antibióticos y la decisión de si serán combinados o no deberá estar adaptada a los datos de sensibilidad de los organismos ${ }^{20}$.

Se analizó la ocurrencia de la contaminación de superficies inanimadas en ambientes hospitalarios, se ha encontrado la presencia de estos agentes patógenos en superficies que son mayormente manipuladas por pacientes $y$ 
personal en cuyos resultados sale de manifiesto la presencia de $\mathcal{P}$. aeruginosa, $C$. difficile, $\mathcal{A}$. baumanni y $S$. aereus resistente a la meticilina; sin embargo más frecuentemente este último en camas de los pacientes y $\mathcal{P}$. aeruginosa con mayor prevalencia en grifos ${ }^{21}$, lo que sugiere que usualmente lugares considerados limpios, a menudo son ignorados lo que facilita su diseminación; además de que en lo que respecta a $\mathscr{P}$. aeruginosa como sugiere nuestro estudio, no solamente puede hallarse en lugares húmedos esto debido a que es un patógeno ubicuo con un requerimiento nutricional mínimo ${ }^{22}$.

Según resultados obtenidos en el Hospital Viedma el 2015, se encontró el crecimiento de bacilos gran negativos de la familia Enterobacteriaceae como $\mathcal{K}$. pneumoniae, de las muestras recogidas de los carritos de curación ${ }^{23}$, resultado diferente al encontrado en nuestro estudio.

En algunos Hospitales de Santiago de Cali, ciudad de Colombia se realizaron alrededor de 123.798 aislamientos, donde representaron el $20 \%$ de muertes en dicha región, vale mencionar que aproximadamente un $65 \%$ de estos aislamientos, pertenecen a Enterobacterias, con predominancia de $\mathcal{E}$. coli, $\mathcal{K}$. pneumoniae y $S$. aereus en sus muestras aisladas ${ }^{24}$, a diferencia de los resultados obtenidos en las superficies del Hospital Solomon Klein en el que prevalece $\mathrm{H}$. alvei.

La presente investigación revelo la presencia de cinco tipo de bacterias, entre ellas $\mathscr{P}$. aeruginosa, $\mathcal{H}$. alvei, $\mathcal{E}$. coli, $\mathcal{K}$. aerogenes y $\mathcal{K}$. pneumoniae, demostrando de esta manera, una alta presencia de cepas Hafnia alvei que se aproxima al $50 \%$ de las muestras aisladas en el Hospital Solomon Klein, seguido de la bacteria E. coli y una cantidad importante de la bacteria Pseudomona aeruginosa, en base a estos resultados el antibiograma descarta la presencia de cepas BLEE en inmediaciones del Hospital Solomon Klein.

\section{CONCLUSIÓN}

Con la presente investigación se pudo evidenciar y constatar la presencia de Pseudomona aeruginosa y al momento de realizar el antibiograma con dichas muestras positivas, se determinó su sensibilidad a los fármacos de elección para dicha bacteria, a la vez se descartó la presencia de cepas BLEE en inmediaciones del Hospital Solomon Klein.

\section{Limitaciones del estudio:}

El cultivo de las cepas BLEE pudo verse limitada por una preparación carente de nutrientes, limitación en el cultivo o manejos laboratoriales de los medios de cultivo.

Agradecimientos sinceros a la Dra. Cecilia Negrón (directora del Hospital Solomon Klein), Dra. Rosario Severich (jefa de laboratorio del Hospital Solomon Klein) y el Dr. Gumersindo Ledo (jefe médico del Hospital Solomon Klein) por la colaboración brindada en la realización del trabajo.

\section{Conflicto de Intereses}

Los autores expresan que no hubo un conflicto de intereses en la realización de este trabajo de investigación.

\section{REFERENCIAS}

1.- Tafur JD, Torres JA y Villegas MV. Mecanismos de resistencia a los antibioticos en bacterias gram negativas; CIDEIM [Internet] 2008; [citado el 8 de septiembre 2019]; 12 (3): 217-226. Disponible en: http://www. sld.cu/galerias/pdf/sitios/apua-cuba/ mecanismos_de_resistencia_a_los_antibioticos_en_ bacterias_gram_negativas.pdf

2.- Roca Lujan A. Pseudomonas aeruginosa un adversario peligroso; Acta Bioquím Clín Latinoam [Internet] 2014; [Citado el 8 de septiembre de 2019]; 48 (4): 465-74 Disponible en: https://www.redalyc.org/ pdf/535/53535594009.pdf

3.- Murray P, Rosenthal K, Pfaller M. Pseudomonas y bacterias relacionadas, Murray $\mathrm{P}$, Rosenthal $\mathrm{K}$ Pfaller M; Microbiología Médica; 8va Éd. Barcelona: El Servier; p.272-279
4.- Hernández A, Gomez J. Infecciones nosocomiales por Pseudomonas aeruginosa multiresistente incluido carbapenémicos: factores predictivos y pronósticos. Estudio prospectivo 2016-2017. Rev Esp Quimioter. [internet] 2018 [Citado el 8 de septiembre de 2019] 31(2): 123-130. Disponible en: https://www. ncbi.nlm.nif.gov/pmc/articles/PMC6159385/

5.-Falconí Sarmiento A, Nolasco Mejia M, Bedoya Rozas A, Amaro Giraldo C, Málaga G. Frecuencia y factores de riesgo para bacteriemia por enterobacterias productoras de betalactamasa de espectro extendido en pacientes de un hospital público de Lima, Perú; Rev Peru Med Exp Salud Publica; [internet] 2018 [citado el 8 de septiembre de 2019] 35 (1): 62-67. Disponible en: https://www. scielosp.org/article/rpmesp/2018.035n1/62-67/ 
6.- Rojas L, Salazar T, Salazar A. Prevalencia de Enterobacterias en Grifos y Lavabos en el Servicio de Medicina Interna del Hospital Obrero №1. SCientifica [Internet] 2009 [citado el 13 de septiembre de 2019]: 7(1): 33-36. Disponible en: https:/imbiomed.com. mx/1/1/ articulos.php? method $=$ showDetaile 2 id_articulo $=65007 \mathrm{R}$ ¿ id seccion $=3537$ QZid_ejemplar $=6534$ QZid_revista $=211$

7.- Fariñas C, Martínez $L_{i}$ Infecciones causadas por bacterias gramnegativas multirresistentes: enterobacterias, Pseudomonas aeruginosa, Acinetobacter baumannii y otros bacilos gramnegativos no fermentadores; ElSevier [Internet]. 2013 [citado el 8 de septiembre de 2019]; 31(6): 402-409. Disponible en: https://www.elsevier.es/esrevista-enfermedades-infecciosas-microbiologia-clinica-28articulo-infecciones-causadas-por-6acterias-gramnegativasS0213005X13000955

8.- Tapia J, Gonzales A, Zeballos J,Crespo, Dra. Santa Cruz A. Identificación y antibiograma de P. aeruginosa en el Pabellon Infantil de Quemados del Hospital Viedma, en agosto del 2013. Rev Cient Cien Med [Internet] 2014. [Citadoel 22 de agosto del 2019] 17(1): 19-22. Disponible en: http:/www.scielo.org.6o/scielo. php? script $=$ sci_arttextcL $p i d=S 1817-74332014000100006$

9.- Apezteguía L, Giachetto G. Ectima gangrenoso y osteoartritis por Pseudomonas aerugínosa en un paciente previamente sano. Arch. Pediatr. Urug. Internet] 2013 [Citado el 22 de agosto de 2019]; 84(2): 127-131. Disponible en: http://www.scielo.edu.uy/scielo. php? script $=$ sci_arttextetpid $=S 1688-12492013000200008$

10.- Dra. Coralith G.; Infecciones por Enterobacterias productoras de $\beta$-lactamasas de espectro extendido; Rev Med Hered [Internet] 2013 [Citado el 8 de septiembre] 24(2): 99-100. Disponible en: http://www. scielo. org.pe/scielo. php? script $=$ sci_arttext LIpid=S1018130X2013000200001

11.- Cabrera C, M.SC, Gómez R, Zuñiga A. La resistencia de bacterias a antibiotioticos, antisépticos y desinfectantes una manifestación de los mecanismos de supervivencia y adaptación. Colomb Med [Internet] 2009 [citado el 8 de septiembre de 2019] 38(2) 149-158. Disponible en:

12.-RamosY,Guillermina A.Evaluación dela resistencia a agentes desinfectantes de bacterias aisladas de ambientes naturales Rev. Soc. Ven. Microbiol. [Internet] 2011 [Citado el 8 de septiembre de 2019] 31(2): 130-137. Disponible en: http://ve.scielo.org/scielo. php? script $=$ sci arttexteI pid $=S 1315-25562011000200009$

13.- Rivera Jacinto $M$, Rodríguez Ulloa $C$, Huayán Dávila G, Mercado-Martínez P. Susceptibilidad a betalactámicos y resistencia por betalactamasas de espectro extendido (BLEE) en Enterobacteriaceae aisladas de reservorios ambientales de un Hospital General en Cajamarca, Perú; Rev Med Hered. [Internet] 2011 [Citado el 8 de Septiembre de 2019]; 22(2): 69-75. Disponible en: http://wrurw scielo org pe/scielo. php? script $=$ sci_arttext\&2pid $=S 1018-130 \times 2011000200005$

14.-Marcano D, De Jesús A, Hernández L, Torres L. Frecuencia de enzimas asociadas a sensibilidad disminuida a betalactámicos en aislados de enterobacterias, Caracas, Venezuela; Rev Panamerican Salud Pública [Internet] 2011 [Citado el 8 de septiembre 2019]; 30(6) 529-534. Disponible en: $\quad h t t p s: / / w w w . s c i e l o s p . o r g / s c i e l o . p h p ?$ pid=S102049892011001200005 \&'script $=$ sci_arttexteZ $t$ ing $=p$

15.- Fari C, Martínez Martínez L. María C.F., Luis M.M. Infecciones causadas por bacterias gramnegativas multirresistentes: enterobacterias, Pseudomonas aeruginosa, Acinetobacter baumannii $y$ otros bacilos gramnegativos no fermentadores; Enferm
Infecc Microbiol Clin. [Internet] 2013 [Citado el 8 de septiembre de 2019] 31(6):402-409. Disponible en: https://www.seimc.org/contenidos/documentoscientificos/ eimc/seimc_eimc_v31n06p402a409.pdf

16.-García Hernández A, García Vázquez E, Hernández Torres A, Ruiz J, Yagüe G, Herrero J, et al. Bacteriemias por Escherichia coli productor de betalactamasas de espectro extendido (BLEE): significación clínica y perspectivas actuales; Rev Esp Quimioter [Internet] 2011 [Citado el 8 de septiembre de 2019]; 24(2):5766. Disponible en: https://seq.es/seq/0214-3429/24/2/ garcia.pdf

17.-Perez Montoya L, Zurita Villarroel I, Pérez Rojas $\mathrm{N}$, Patiño Cabrera $\mathrm{N}$, Calvimonte O. Ifecciones intrahospitalarias: Agentes, manejo actual y prevención. Rev Cient Cienc Méd ['nternet] 2010 [Citado el 22 de septiembre de 2019]; 13(2): 9094. Disponible en: http://www.scielo.org.6o/scielo. php? pid $=$ S1817-74332010000200009\&'script $=$ sci_arttext

18.-Delpiano Méndez L. Infecciones cruzadas en las prácticas de salud ambulatorias Medwave [Internet] 2009 [Citado el 23 de abril de 2020]; 9(6):e3987. Disponible en: https://www.medwave.cl//ink.cgi/ Medwave/PuestaDia/APS/3987

19.- Anton Y. Peleg, Hooper C. Infecciones intrahospitalarias por bacterias gram-negativas. Engl J Med [lnternet] 2010 [Citado el 22 de septiembre]. 362:1804-13. Disponible en: http://www.intramed.net/ contenidover.asp? contenidoI $\mathcal{D}=65629$

20.-Jiménez A González del Castillo, Martínez Ortiz de Zárate J. M, Candel González F, Piñera salmerón P, Moya Mir M.S. Características y cambios epidemiológicos de los pacientes con Neumonía adquirida en la comunidad en los servicios de urgencia hospitalarios. Rev. An. Sist. Sanit. Navar. [Internet] 2013 [Citado el 22 de septiembre de 2019]; 36(3): 380-95. Disponible en: http://scielo.isciii.es/pdff/ asisna/v36n3/04_original.pdf

21.- Oliveira, Damasceno A, Souza Q. Superficies inanimadas del ambiente hospitalario como posibles reservorios de bacterias resistentes: una revisión. Rev. esc. enferm. USP [Internet] 2010 [Citado el 23 de abril de 2020]; 44(4): 1118-1123. Disponible en: $\quad h t t p: / / w w w . s c i e l o .6 r / s c i e l o . p h p ?$ pid=S008062342010000400038 \& script $=$ sci_abstract 2 Zting $=e s$

22-Paz-Zarza VM, Mangwani-Mordani S, MartínezMaldonado A, Álvarez-Hernández D, Solano-Gálvez SG, Vázquez-López R. Pseudomonas aeruginosa: patogenicidad y resistencia antimicrobiana en la infección urinaria. Rev. chil. Infectol., [Internet] 2019 [Citado el 23 de abril del 2020]; 36(2) 180189. Disponible en: https://scielo.conicyt.cl/scielo. php? script $=$ sci_arttextcLid $=$ S0716-10182019000200180

23.- Becerra Torrejón DJ, Almanza Salinas GG, Flores Alarcón AA, Santa Cruz A. Bacterias en tapas de antisépticos y pinzas de traspaso en carros de curación de emergencias, Hospital Clínico Viedma 2015. Rev Cient Cienc Méd [Internet] 2016 [Citado el 22 de septiembre de 2019]; 19(1): 17-21. Disponible en: $\quad h t t p: / / w w w$. scielo.org.6o/scielo.php? script=sci arttexteLpid $=$ S1817-74332016000100004

24.- Martinez Buitrago E, Hernández C, Pallares C, Pacheco R, Hurtado K; Frecuencia de aislamientos microbiológicos y perfil de resistencia bacteriana en 13 clinicas y hospitales de alta complejidad en Santiago de Cali-Colombia. Infect., Bogotá [Internet] 2014 [Citado el 23 de abril de 2020]; 18(1):3-11. Disponible en: https://www.sciencedirect.com/science/ article/pii/S0123939214707349 